\title{
Correction to: Vacuum Circuit Breaker for Aviation Variable Frequency Power System
}

\section{Correction to:}

Y. Jiang and Q. Li, Vacuum Circuit Breaker for Aviation

Variable Frequency Power System, Power Systems

https://doi.org/10.1007/978-981-33-4781-6

In the original version of the book, the following information has been added: Funding

details have been added to the Preface section. 